\title{
Supramolecular nano-assemblies with tailorable surfaces: recyclable hard templates for engineering hollow nanocatalysts
}

\author{
Li-Na Han, Tian-Nan Ye, Li-Bing Lv, Kai-Xue Wang, Xiao Wei, Xin-Hao Li* and Jie-Sheng Chen*
}

\begin{abstract}
Supramolecular assemblies are introduced here as new-concept hard templates for the synthesis of hollow nanostructures (exemplified with $\mathrm{TiO}_{2}$ hollow nanostructures in this work). Supramolecular templates with tunable morphology and rich surface functional groups facilitate the tight coating of other materials for the formation of hollow nanostructures. The weak interaction between the supramolecules or micromolecules benefits the facile removal of the templates for large-scale synthesis of hollow nanostructures and also affords excellent template reusability. This method allows for the incorporation of various metal dopants into the $\mathrm{TiO}_{2}$ lattice, as a typical example of nanocatalyst, by introducing the corresponding metal salt as a dopant source. High-resolution transmission electron microscopy (HRTEM), X-ray diffraction (XRD) and UV-vis absorption spectroscopy investigations suggested substitution of $\mathrm{Ti}^{4+}$ sites by $\mathrm{Co}^{2+}$, which increased the activity of the catalytic sites in the doped materials, reducing the overpotential of $\mathrm{TiO}_{2}$ for the oxygen evolution reaction.
\end{abstract}

The synthesis of hollow micro- and nanoscale structures has attracted great interest owing to their high surface areas, low density, and unique optical and electronic features $[1,2]$. All of these features make them suitable for a variety of applications including catalysis [3], solar cells [4,5], lithium-ion batteries [6], optical imaging [7], and drug delivery [8]. Especially for their applications in catalysis and photocatalysis, hollow micro- and nanoscale structures can provide a large active surface area, reduced diffusion resistance, excellent mass transfer efficiency, and high dispersity in reaction media with excellent reusability. The void space can also act as a catalyst container for embedded fine metal nanoparticles to extend their life-time $[9,10]$. Moreover, the thin shell of hollow micro- and nanoscale structures can facilitate charge transfer to surface active sites [11], which implies that they have great potential as highly efficient photocatalysts for water splitting and artificial photosynthesis [12].

Although great efforts have been made towards the development of template-free routes for the preparation of hollow structures [13-15], template-directed synthet- ic methods have been established as one of the most effective and controllable methods for synthesizing hollow structures [16]. The most commonly used templates can broadly be divided into two categories: hard templates and soft templates. Monodisperse silica, polymers, carbon nanospheres, and nanoparticles of metals and metal oxides have been used as hard templates for preparation of hollow structures [17-20]. The synthesis of designed materials with hollow structures requires the templates to have additional surface functionalization. Because of droplet coalescence and Ostwald ripening, it is challenging to obtain uniform and small structures using soft templates (such as emulsion droplets, latexes, and gas bubbles) [21-23]. The hard and soft templates used previously could be considered sacrificial templates. Tedious post-treatment processes (e.g., calcination at high temperature, chemical etching) are usually used to remove such templates [19,24]. All these processes significantly increase the final cost of the hollow nanostructures in large-scale applications. Consequently, recyclable templates with controlled morphology and surface functional groups are thus highly desirable to fabricate hollow nanostructures in large quantities, and also to further lower their cost for practical applications that so far have been well defined only at the lab scale $[25,26]$.

As an alternative, nano-assemblies of supramolecules or macromolecules offer a number of advantages, and show great potential as nanotemplates. Surfaces with rich surficial functional groups facilitate their tight binding with other materials to form nanostructures [27]. Controlled noncovalent assembly of certain small molecules in the presence of specific inorganic components enables the formation of ordered nanostructures with well-defined size and shape. Weak interactions among the supramolecules or micromolecules allow the facile removal of the templates to allow the large-scale synthesis of hollow nanostructures and afford excellent reusability of the templates. As described in the literature, such supramolecules or macromolecules are usually applied as soft templates for the for- 
mation of porous nanostructures or thin films [28].

Herein, we describe the application of the "hard" aspect of supramolecular nanoassemblies for the synthesis of hollow nanostructures (exemplified with $\mathrm{TiO}_{2}$ here). Both the size and thickness of the hollow nanostructures on the surface of the supramolecular nanotemplates were tuned. This method also allowed for facile incorporation of various metal dopants to significantly elevate the activity of the catalytic sites in the doped material.

A cyanuric acid-melamine complex was chosen as the possible supramolecular nanotemplate owing to the ease of control of its morphology and rigid structure $[23,29]$. Recently, flower-like assemblies of melamine and cyanuric acid were synthesized by Jun et al. [30] and Shalom et al. [31] for further thermal condensation into porous carbon nitride nanostructures, where the rigid structures of the nanoflowers affected the morphology of the carbon nitride nanostructures and thus their photocatalytic activities. This observation suggested the mechanical stability of these cyanuric acid-melamine assemblies even at elevated temperature. Moreover, the $-\mathrm{NH}_{2}$ groups of melamine and the $-\mathrm{OH}$ groups of cyanuric acid also make it possible to change the surficial functional groups of the as-formed cyanuric acid-melamine assemblies for specific purposes by varying the ratio of cyanuric acid to melamine (Fig. 1). Also, cyanuric acid-melamine assemblies can be dissolved into an excess amount of water or hot water (around $80^{\circ} \mathrm{C}$ ). This implies that the sustainable removal and reuse of the cyanuric acid-melamine assemblies is possible. All of these features provide cyanuric acid-melamine assemblies with great potential as a recyclable hard template for the synthesis of hollow nanostructures.

Cyanuric acid-melamine nanoassemblies can be facilely tuned to control their morphology. Many different ordered textures of the cyanuric acid-melamine complex can thus be obtained, depending on the solvent in which it is

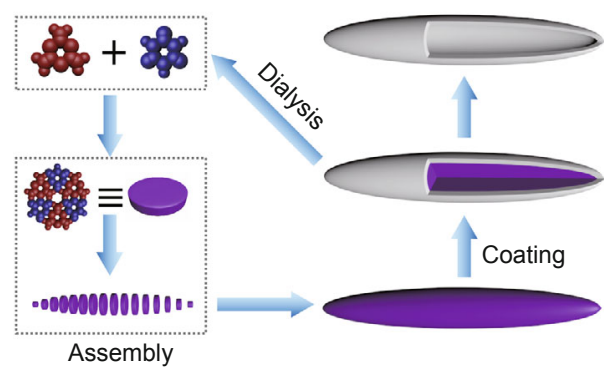

Figure 1 Schematic mechanism illustrating the use of supramolecular assemblies (purple) of cyanuric acid (red) and melamine (blue) as recyclable hard templates for hollow structures (white) via a three-step strategy: (1) formation of the cyanuric acid-melamine nanotemplate via supramolecular assembly, (2) coating of $\mathrm{TiO}_{2}$ on the template, and (3) removal and recycling of the template via dialysis in an excess amount of water. mixed. Both the shape and size of the present cyanuric acid-melamine assemblies were controlled by using different solvents (Table S1 and Fig. S1) or tuning the concentrations and weight ratios of the melamine and cyanuric acid (Fig. S2). Taking the ellipsoid-like cyanuric acid-melamine nanorods (e-CM-NRs) as an example, both the length and the diameter (the maximum diameter at the central part) can be tuned within a quite broad range simply by altering the concentration of melamine and cyanuric acid (Figs S3a-d). The synthetic method is rather facile. Uniform e-CM-NRs, as revealed by the scanning electron microscopy (SEM) observations (Fig. S3a), were obtained in large quantities (Fig. S4) simply by mixing aqueous solutions of melamine and cyanuric acid, promising a great potential as abundant and recyclable templates for large-scale synthesis of hollow nanostructures.

For practical application of nanoassemblies as templates, a tailorable surface with rich functional groups is the key parameter for achieving better coating with additional shells. The present cyanuric acid-melamine nanoassemblies are such a nanotemplate. As shown in Fig. 2a, the zeta potentials of the cyanuric acid-melamine complexes were negative, which is beneficial for forming $\mathrm{TiO}_{2}$ on the surface of the templates. The surface charges of the complexes decreased with increasing mass ratio of melamine and cyanuric acid. The cyanuric acid-melamine complexes (Fig. 2b) with a mass ratio of 4:5 were selected as new-concept hard templates for replicating $\mathrm{TiO}_{2}$ hollow nanostructures owing to their highly charged surface. The coating
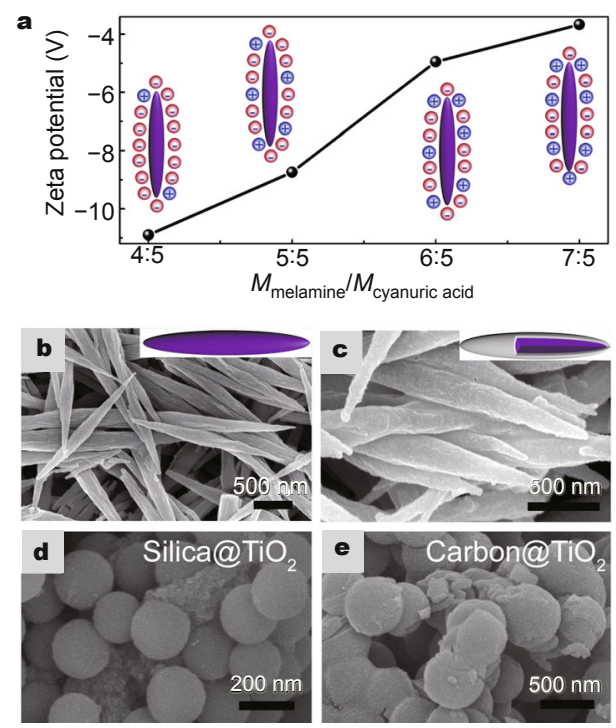

Figure 2 (a) Zeta potentials of cyanuric acid-melamine nanotemplates modulated by varying the weight ratios of melamine and cyanuric acid. SEM images of cyanuric acid-melamine nanotemplates (b), $\mathrm{TiO}_{2}$ coated cyanuric acid-melamine templates (c), $\mathrm{SiO}_{2}$ spheres (d), and carbon spheres (e) obtained under the same conditions. 
of $\mathrm{TiO}_{2}$ on these cyanuric acid-melamine nanotemplates was perfect (Fig. 2c), without the need for additional surface functionalization. Both the shape and the size of the as-obtained $\mathrm{TiO}_{2}$ nanostructures were controlled simply by choosing different nanotemplates (Fig. S5). Taking e-CMNRs as the example, the morphology of the e-CM-NRs was not obviously changed after coating with a $\mathrm{TiO}_{2}$ layer (Figs $\mathrm{S} 3 \mathrm{e}-\mathrm{h}$ and $\mathrm{S} 6$ ). Uniform $\mathrm{TiO}_{2}$ hollow nanostructures were obtained after the removal of e-CM-NRs (Figs S3i-l). Note that the silica and carbon nanospheres without surface modification (Fig. S7) only led to the formation of iregular aggregates (Fig. 2d) or cracked shells (Fig. 2e), confirming the importance of surface modification for better coating with shell materials.

$\mathrm{TiO}_{2}$ nanostructures have been widely investigated as photocatalysts or electrocatalysts for the (photo-) electrolysis of water [32,33]. However, such water splitting reactions are tremendously hindered by the sluggish oxygen evolution reaction (OER) in which water is oxidized to $\mathrm{O}_{2}$ via a 4-electron path [34]. Moreover, the high overpotential and low efficiency of current catalysts and photocatalysts for OER remains a bottleneck in their practical application [35]. Many efforts have thus been devoted to increasing the charge transfer efficiency and lowering the overpotential of various catalysts, including $\mathrm{TiO}_{2}$ based nanocatalysts, to elevate their final OER activity [36]. Among the strategies described in the literature, fine control of the diameter or shell thickness of the $\mathrm{TiO}_{2}$ nanostructures is a direct method of increasing the rate of charge transfer for higher OER activity. The optimal size of a semiconducting nanostructure is related to the mean length of the free migration path for charge carriers, which is generally expected to be in the range of $10-30 \mathrm{~nm}[37,38]$.

Again, the present cyanuric acid-melamine nanoassemblies with amino-group-rich surfaces showed obvious advantages over conventional templates for casting hollow nanostructures with ultra-thin and tunable shells. We therefore initially tuned the thickness of the $\mathrm{TiO}_{2}$ hollow nanostructures to optimize their OER activities. The typical TEM images of the $\mathrm{TiO}_{2}$ hollow nanostructures in Figs $3 \mathrm{a}-\mathrm{c}$ indicated their tunable thickness and uniform morphology (Fig. S8). The thicknesses of the samples were varied from 13 to 23 and $34 \mathrm{~nm}$ simply by changing the amount of tetrabutyl titanate during the coating process (Table S2). The gradual broadening of the optical band gap of these samples was also revealed by the obvious blue-shift of the absorption edge in the UV-vis spectra of the $\mathrm{TiO}_{2}$ hollow nanostructures with decreased thickness (Fig. S9). To appraise the effect of the hollow nanostructure on the OER activity of the $\mathrm{TiO}_{2}$ hollow nanostructures, their OER activity and that of a control sample (P25) were tested using glassy carbon electrodes in $1 \mathrm{M} \mathrm{KOH}$ electrolyte. Fig. 3d shows the linear sweep voltammetry (LSV) curves of all of the tested samples, and reveals the much lower overpotentials of all of the $\mathrm{TiO}_{2}$ hollow nanostructure samples compared with that of $\mathrm{P} 25$. The $\mathrm{TiO}_{2}$ hollow nanostructures with a thickness of $23 \mathrm{~nm}$ (sample $\mathrm{TiO}_{2}-23 \mathrm{~nm}$ in Fig. 3d) exhibited the lowest overpotential among the obtained $\mathrm{TiO}_{2}$ hollow nanostructure samples. Thicker $\mathrm{TiO}_{2}$ nano-capsules have higher valence band position and thus lower potential for water oxidation. Additionally, charge transfer may have been blocked by the thick walls of the hollow structures owing to the low conductivity of $\mathrm{TiO}_{2}$. As a result, the sample $\mathrm{TiO}_{2}-23 \mathrm{~nm}$, which had an effective balance between these two counteracting effects, was selected as the best nanocatalyst for all following experiments.

Owing to the facile synthesis of the present supramolecular nanotemplates for large-scale synthesis of $\mathrm{TiO}_{2}$ hollow nanostructures in water, it was also possible to introduce transition metal dopants into the crystal lattice of $\mathrm{TiO}_{2}$ to enhance their practical application in OER (Figs S10 and S11b). The involvement of transition metal dopants may create additional sub-band gaps in the electronic structure of $\mathrm{TiO}_{2}$ and thereby further enhance its photocatalytic activity and lower its potential for OER [39]. The successful introduction of transition metal dopants can be directly observed from the color change of the $\mathrm{TiO}_{2}$ from white to a variety of colors, depending on the metal dopants introduced. UV-vis spectra (Fig. S11a) indicated that doped $\mathrm{TiO}_{2}$ samples extended their absorption range from

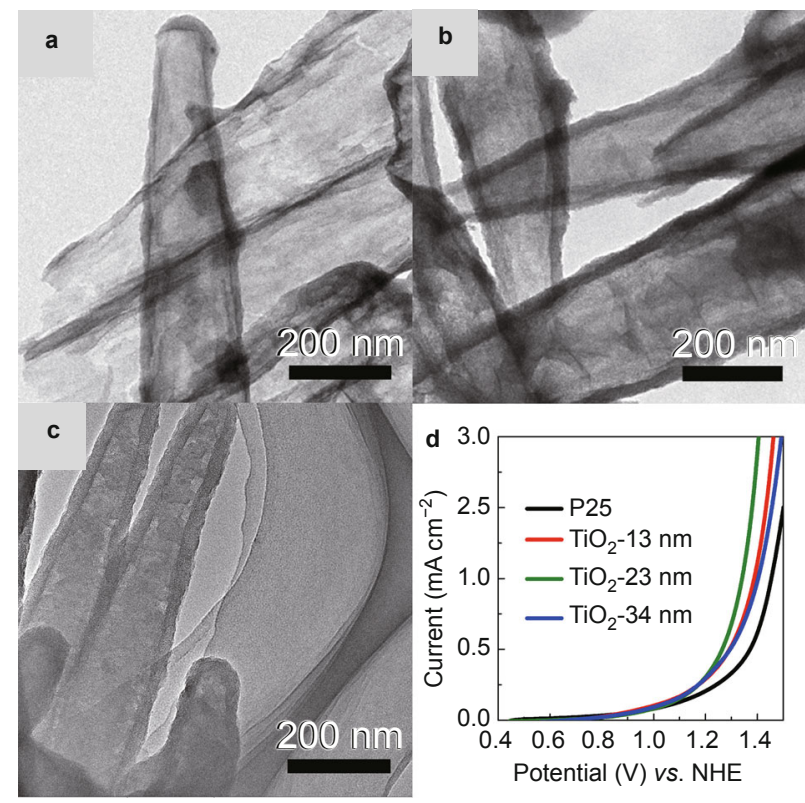

Figure 3 TEM images $\left(\mathrm{a}-\mathrm{c}\right.$ ) and OER activities (d) of $\mathrm{TiO}_{2}$ hollow nanostructures of different thickness: (a) $13 \mathrm{~nm}$, (b) $23 \mathrm{~nm}$, and (c) $34 \mathrm{~nm}$. LSV curves were measured using glassy carbon electrodes (loading catalyst: $0.336 \mathrm{mg} \mathrm{cm}^{-2}$ ) in $1 \mathrm{M} \mathrm{KOH}$ electrolyte. 
ultraviolet to visible light, beneficial for their possible applications in both photocatalytic reactions and OER (Fig. S11b). Indeed, the introduction of transition metal dopants obviously decreased the overpotentials for OER in the sequence Co- $\mathrm{TiO}_{2}<<\mathrm{Ni}-\mathrm{TiO}_{2}<\mathrm{Mn}-\mathrm{TiO}_{2}<\mathrm{V}-\mathrm{TiO}_{2}<\mathrm{Mo}-$ $\mathrm{TiO}_{2}<\mathrm{TiO}_{2}$.

Because the crystallinity of $\mathrm{TiO}_{2}$ nanocatalysts can also dominate their intrinsic electronic structure and thus their overpotential for OER, the as-formed amorphous samples were heated at $400^{\circ} \mathrm{C}$ in air. After calcination, both pristine and doped pure anatase samples (Fig. S12) were obtained with well-preserved uniform hollow structures (Figs $4 \mathrm{a}-\mathrm{b}$ and S13). This indicated that the dopants did not disturb the crystal structure of the $\mathrm{TiO}_{2}$ hollow nanostructures, but only created more defects, as revealed by the HRTEM images of the pristine and doped samples (Figs $4 \mathrm{a}-\mathrm{b}$ and S14) and also their sub-band gap (Fig. S15). Although a high concentration of metal dopant (5 at\%) was introduced, no obvious signals of other metal oxides were detected in the XRD patterns of these samples (Fig. S12a). For Co- and V-doped samples, an obvious shift of the (101) peaks of anatase phase to high angles was observed (Fig. S12b). These results suggested the homogeneous distribution of these metal dopants, which was further confirmed by the elemental mapping results (Fig. 4c, exemplified with Co here). The overpotential of the $\mathrm{Co}-\mathrm{TiO}_{2}$ sample was obviously improved after calcination, indicating the positive effect of increased crystallinity of the $\mathrm{TiO}_{2}$ nanocatalysts on their OER activity (Fig. S16). Moreover, the surface area and pore size of $\mathrm{Co}-\mathrm{TiO}_{2}-400$ were similar to those of the pristine $\mathrm{TiO}_{2}-400$ sample (Fig. S17), which excludes any effect of the mesostructure on the OER activity. Comparing the overpotentials at a specific current density of $0.5 \mathrm{~mA}$ $\mathrm{cm}^{-2}$, the overpotential of $\mathrm{Co}-\mathrm{TiO}_{2}-400$ was estimated to
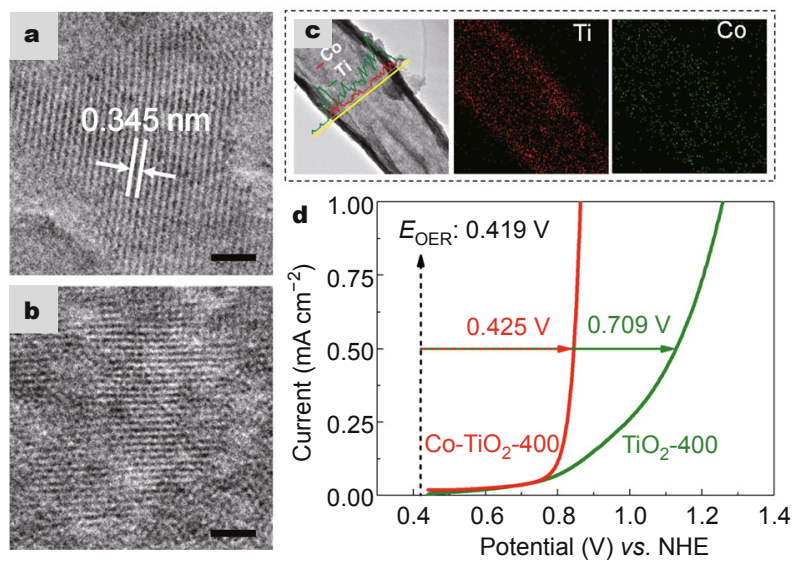

Figure 4 HRTEM (scale bar: $2 \mathrm{~nm}$ ) images of (a) $\mathrm{TiO}_{2}-400$ and (b) Co$\mathrm{TiO}_{2}-400$ hollow nanostructures (obtained via calcination at $400^{\circ} \mathrm{C}$ in air); (c) elemental mapping images of $\mathrm{Co}^{-} \mathrm{TiO}_{2}-400$ hollow nanostructures; (d) LSV curves of the samples for OER. be $0.425 \mathrm{~V} v s$. NHE, much lower than that of pristine $\mathrm{TiO}_{2}-$ 400 (Fig. 4d). This result is much lower than that reported for benchmark $\mathrm{TiO}_{2}$-based nanocatalysts in the literature (0.488 V vs. NHE) [40]. The fact that metal-doped $\mathrm{TiO}_{2}$ hollow nanostructures were successfully prepared suggests the good compatibility of the supramolecular templates with various metal salts.

In conclusion, supramolecular templates with tunable morphology and rich surface functional groups facilitated the tight coating of other materials and allowed easy control of both the shape and size of the resulting hollow nanostructures (exemplified with $\mathrm{TiO}_{2}$ here). The flexibility of the present supramolecular template-based synthetic method allowed us to further tune the electronic structure of the final nanostructure for better OER by engineering its mesostructure and heteroatom doping. More importantly, the simple synthetic method and low cost of the precursors make the present recyclable supramolecular templates suitable for mass production of various hollow nanomaterials in the future. Our work opens up new possibilities for the use of supramolecular assemblies as sustainable hard templates to fabricate novel hollow nanostructures which can function as efficient nanocatalysts, photocatalysts, electrode materials, and drug-carriers for practical use.

Received 27 October 2014; accepted 4 December 2014; published online 22 December 2014

1 Sieb NR, Wu NC, Majidi E, et al. Hollow metal nanorods with tunable dimensions, porosity, and photonic properties. ACS Nano, 2009, 3: 1365-1372

2 Lou XW, Archer LA, Yang Z. Hollow micro-/nanostructures: synthesis and applications. Adv Mater, 2008, 20: 3987-4019

3 Bai F, Sun Z, Wu H, et al. Templated photocatalytic synthesis of well-defined platinum hollow nanostructures with enhanced catalytic performance for methanol oxidation. Nano Lett, 2011, 11: 3759-3762

4 Bao CX, Huang H, Yang J, et al. The maximum limiting performance improved counter electrode based on a porous fluorine doped tin oxide conductive framework for dye-sensitized solar cells. Nanoscale, 2013, 5: 4951-4957

5 Wu X, Lu GQ, Wang L. Dual-functional upconverter-doped $\mathrm{TiO}_{2}$ hollow shells for light scattering and near-infrared sunlight harvesting in dye-sensitized solar cells. Adv Energy Mater, 2013, 3: 704-707

6 Yao Y, McDowell MT, Ryu I, et al. Interconnected silicon hollow nanospheres for lithium-ion battery anodes with long cycle life. Nano Lett, 2011, 11: 2949-2954

7 Wu H, Zhang S, Zhang J, et al. A hollow-core, magnetic, and mesoporous double-shell nanostructure: in situ decomposition/reduction synthesis, bioimaging, and drug-delivery properties. Adv Funct Mater, 2011, 21: 1850-1862

8 Wei W, Ma GH, Hu G, et al. Preparation of hierarchical hollow $\mathrm{CaCO}_{3}$ particles and the application as anticancer drug carrier. J Am Chem Soc, 2008, 130: 15808-15810

9 Wang D, Hisatomi T, Takata T, et al. Core/shell photocatalyst with spatially separated Co-catalysts for efficient reduction and oxidation of water. Angew Chem Int Ed, 2013, 52: 11252-11256 
10 Liu R, Mahurin SM, Li C, et al. Dopamine as a carbon source: the controlled synthesis of hollow carbon spheres and yolk-structured carbon nanocomposites. Angew Chem Int Ed, 2011, 50, 6799-6802

11 Okada T, Miyamoto K, Sakai T, Mishima S. Encapsulation of a polyoxometalate into an organosilica microcapsule for highly active solid acid catalysis. ACS Catal, 2013, 4: 73-78

12 Ye TN, Xu M, Fu W, et al. The crystallinity effect of mesocrystalline $\mathrm{BaZrO}_{3}$ hollow nanospheres on charge separation for photocatalysis. Chem Commun, 2014, 50: 3021-3023

13 Jana S, Chang JW, Rioux RM. Synthesis and modeling of hollow intermetallic Ni-Zn nanoparticles formed by the Kirkendall effect. Nano Lett, 2013, 13: 3618-3625

14 Han FD, Bai YJ, Liu R, et al. Template-free synthesis of interconnected hollow carbon nanospheres for high-performance anode material in lithium-ion batteries. Adv Energy Mater, 2011, 1: 798-801

15 Wang Z, Zhou L, Lou XW. Metal oxide hollow nanostructures for lithium-ion batteries. Adv Mater, 2012, 24: 1903-1911

16 Li XH, Zhang DH, Chen, JS. Synthesis of amphiphilic superparamagnetic ferrite/block copolymer hollow submicrospheres. J Am Chem Soc, 2006, 128: 8382-8383

17 Qiao ZA, Guo B, Binder AJ, et al. Controlled synthesis of mesoporous carbon nanostructures via a "silica-assisted" strategy. Nano Lett, 2012, 13: 207-213

18 Choi SH, Ankonina G, Youn DY, et al. Hollow ZnO nanofibers fabricated using electrospun polymer templates and their electronic transport properties. ACS Nano, 2009, 3: 2623-2631

19 Liu H, Qu J, Chen Y, et al. Hollow and cage-bell structured nanomaterials of noble metals. J Am Chem Soc, 2012, 134: 11602-11610

20 Ha TL, Kim JG, Kim SM, Lee IS. Reversible and cyclical transformations between solid and hollow nanostructures in confined reactions of manganese oxide and silica within nanosized spheres. J Am Chem Soc, 2012, 135: 1378-1385

21 Wang B, Chen JS, Wu HB, Wang Z, Lou XW. Quasiemulsion-templated formation of $\alpha-\mathrm{Fe}_{2} \mathrm{O}_{3}$ hollow spheres with enhanced lithium storage properties. J Am Chem Soc, 2011, 133: 17146-17148

22 White RJ, Tauer K, Antonietti M, Titirici MM. Functional hollow carbon nanospheres by latex templating. J Am Chem Soc, 2010, 132: $17360-17363$

23 Chen X, Wu Y, Su B, et al. Terminating marine methane bubbles by superhydrophobic sponges. Adv Mater, 2012, 24: 5884-5889

24 Wang Y, Su X, Lu S. Shape-controlled synthesis of $\mathrm{TiO}_{2}$ hollow structures and their application in lithium batteries. J Mater Chem, 2012, 22: 1969-1976

25 Fechler N, Fellinger TP, Antonietti M. "Salt templating": a simple and sustainable pathway toward highly porous functional carbons from ionic liquids. Adv Mater, 2013, 25: 75-79

26 Tian $\mathrm{P}, \mathrm{Ye} \mathrm{J}, \mathrm{Xu} \mathrm{N}$, et al. A magnesium carbonate recyclable template to synthesize micro hollow structures at a large scale. Chem Commun, 2011, 47: 12008-12010

27 Zhou Y, Yan D. Supramolecular self-assembly of amphiphilic hyperbranched polymers at all scales and dimensions: progress, characteristics and perspectives. Chem Commun, 2009, 1172-1188

28 Antonietti M. Silica nanocasting of lyotropic surfactant phases and organized organic matter: material science or an analytical tool? Philos Trans R Soc A-Math Phys Eng Sci, 2006, 364: 2817-2840
29 Mathias JP, Simanek EE, Whitesides GM. Self-assembly through hydrogen bonding: peripheral crowding-a new strategy for the preparation of stable supramolecular aggregates based on parallel, connected $\mathrm{CA}_{3} \cdot \mathrm{M}_{3}$ rosettes. J Am Chem Soc, 1994, 116: 4326-4340

30 Jun YS, Lee EZ, Wang X, et al. From melamine-cyanuric acid supramolecular aggregates to carbon nitride hollow spheres. Adv Funct Mater, 2013, 23: 3661-3667

31 Shalom M, Inal S, Fettkenhauer C, Neher D, Antonietti M. Improving carbon nitride photocatalysis by supramolecular preorganization of monomers. J Am Chem Soc, 2013, 135: 7118

32 Zou XX, Li GD, Guo MY, et al. Heterometal alkoxides as precursors for the preparation of porous $\mathrm{Fe}$ - and $\mathrm{Mn}-\mathrm{TiO}_{2}$ photocatalysts with high efficieccies. Chem Eur J, 2008, 14: 11123-11131

33 Kong M, Li Y, Chen X, et al. Tuning the relative concentration ratio of bulk defects to surface defects in $\mathrm{TiO}_{2}$ nanocrystals leads to high photocatalytic efficiency. J Am Chem Soc, 2011, 133: 16414-16417

34 Li YF, Selloni A. Mechanism and activity of water oxidation on selected surfaces of pure and Fe-doped $\mathrm{NiO}_{x}$. ACS Catal, 2014, 4: $1148-1153$

35 Chen S, Duan J, Jaroniec M, Qiao SZ. Three-dimensional N-doped graphene hydrogel/NiCo double hydroxide electrocatalysts for highly efficient oxygen evolution. Angew Chem, 2013, 125: 13812-13815

36 Li YF, Liu ZP, Liu L, Gao W. Mechanism and activity of photocatalytic oxygen evolution on titania anatase in aqueous surroundings. J Am Chem Soc, 2010, 132: 13008-13015

37 Sun J, Zhang J, Zhang M, et al. Bioinspired hollow semiconductor nanospheres as photosynthetic nanoparticles. Nat Commun, 2012, 1139

38 Li YF, Liu ZP. Particle size, shape and activity for photocatalysis on titania anatase nanoparticles in aqueous surroundings. J Am Chem Soc, 2011, 133: 15743-15752

39 Pfrommer LJ, Lublow M, Azarpira A, et al. A molecular approach to self-supported cobalt-substituted $\mathrm{ZnO}$ materials as remarkably stable electrocatalysts for water oxidation. Angew Chem Int Ed, 2014, 53: 5183-5187

40 Liu B, Chen HM, Liu C, et al. Large-scale synthesis of transition-metal-doped $\mathrm{TiO}_{2}$ nanowires with controllable overpotential. J Am Chem Soc, 2013, 135: 9995-9998

Acknowledgements This work was financially supported by the National Basic Research Program of China (2013CB934102 and 2011CB808703) and the National Natural Science Foundation of China (21331004 and 21301116).

Author contributions $\mathrm{Li} \mathrm{XH}$ and Chen JS designed the experiments, conceived the post-fabrication tuning of random modes; Han LN performed the experiments and the data analysis with help from Ye TN, and wrote the paper with support from Li XH. All authors contributed to the general discussion.

Conflicts of interest The authors declare that they have no conflict of interest.

Supplementary information Experimental details and supplementary data are available in the online version of the paper. 


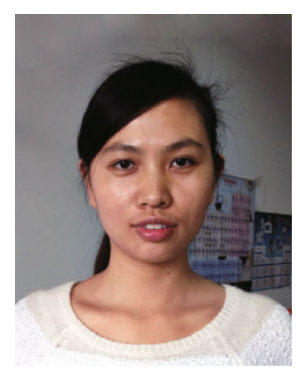

Li-Na Han received her bachelor's degree in chemistry from Jilin University in 2012. She is currently studying for a $\mathrm{PhD}$ at Shanghai Jiao Tong University. Her research interests are based in the study of catalysis using $\mathrm{TiO}_{2}$ nanostructures.

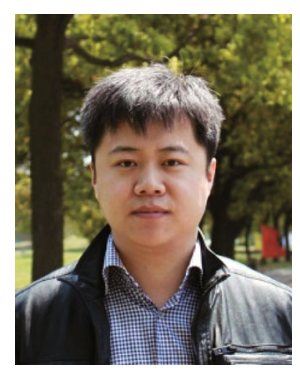

Xin-Hao Li completed each of his academic degrees at the Department of Chemistry of Jilin University from 1999 to 2009, receiving his PhD in 2009 with Professor Jie-Sheng Chen. He then joined Prof. Markus Antonietti's group as an Alexander von Humboldt Research Fellow at the Max-Planck Institute of Colloids and Interfaces from 2009 to 2012. Since 2013, he has been a professor at the School of Chemistry and Chemical Engineering, Shanghai Jiao Tong University. His current scientific interests are mainly focused on the synthesis of carbon nitride and graphene based functional materials for energy and environmental science applications.

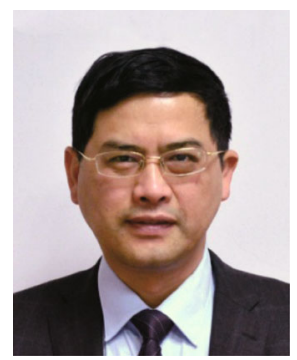

Jie-Sheng Chen received his PhD degree from Jilin University in 1989 and worked as a postdoctoral fellow in the Royal Institution of Great Britain, the United Kingdom, from 1990 to 1994, and as a professor at the Department of Chemistry, Jilin University from 1994 to 2008. Since 2008, he has been a professor at the School of Chemistry and Chemical Engineering, Shanghai Jiao Tong University. His research interests are based in the synthesis of solid compounds and composite materials with new structures and functions.

中文摘要 超分子自组装化合物具有可调控的形貌和丰富的表面官能团. 超分子或小分子单体之间的弱相互作用有利于除去模板, 作 为硬模板合成空心纳米结构 (以 $\mathrm{TiO}_{2}$ 为例), 不仅能得到具有可调控形貌的空心纳米结构, 并且超分子模板可以回收和重复利用. 实验表 明, 用三聚氧氨和三聚氨酸作为原料合成的超分子模板, 有利于在模板表面复合无机组分, 并且模板中的氢键在水中易断裂, 因此可以 通过透析方法除去模板得到空心纳米结构. 这种超分子模板法也可以用于合成掺杂金属离子的空心结构纳米催化剂, 进一步调控电子 结构增加析氧反应的活性位点, 降低超电势. 更重要的是, 此模板合成方法简单、耗能低、可重复利用, 可以用于合成大批量的空心结 构纳米催化剂。 